\title{
AN IMPROVED EMBALMING FLUID FORMULA
}

\author{
RUSSELL T. WOODBURNE AND CARL A. LAWRENCE \\ Departments of Anatomy and Bacteriology, University of Michigan \\ Medical School, Ann Arbor

\section{INTRODUCTION}

Although a local modification of the usual alcohol-glycerinephenol-formaldehyde embalming formula has proved generally satisfactory through the years, continued price advances in the principal items of this formula led to its re-examination from a number of standpoints. A first consideration was the investigation of the possibility of substitutes for those items, such as ethanol and glycerine, used in a number of industrial processes and consequently becoming increasingly costly. Other considerations were the determination of the relative effectiveness of any substitutes utilized and the advisability of incorporating new compounds of possibly greater bactericidal and fungicidal properties. Since glycerine is deemed to contribute to the softness and plasticity of the body by virtue of its ability to hold fluid in the tissues, a product of similar hygroscopic character was indicated as a substitute. Although a number of these are available only two were tested, Aquaresin and Glucarine B, produced by the Glyco Products Company, of Brooklyn, New York. The latter was found to be the better and entirely satisfactory for this purpose. Of the alcohol series, isopropanol seemed to be the logical substitute for ethanol. Isopropanol is readily available and is considered to be a better germicidal and antiseptic agent than ethyl alcohol. Isopropanol has a distinctive odor, but not an objectionable one. The small percentage of formalin in the original formula was thought to be of value and being a relatively inexpensive item was retained. Phenol, having good fungicidal properties, is a worthwhile addition to an em- 
balming formula up to the concentration at which it begins to affect the dissector's hands and is retained here especially because its addition made a formula of greater effectiveness against test-organisms. The quaternary ammonium compounds have been found by a number of investigators to have definite germicidal and fungicidal properties and two of these were used in the in vitro studies as additional elements of the formula. Because of its effectiveness, availability, and reasonable cost, Benzalkonium chloride, chemically known as alkyldimethylbenzylammonium chloride, was chosen for incorporation in the formula and proved a highly desirable addition. Another such compound, Armeen-14D, ${ }^{1}$ was also tested.

Since an embalming fluid must be effective as a preserving agent and must, without possibility of failure, completely protect the dissector from infectious material resident in the specimen, test-tube studies were carried out on a number of infectious organisms and with various modifications of the original formula. The formulas prepared are presented in table 1.

\section{GERMICIDAL TESTS}

I. Germicidal action of embalming solutions on Mycobacterium tuberculosis. All of the formulations given in table 1 were tested against a guinea pig virulent strain of $M$. tuberculosis. The organisms were grown on Petragnani's slants for two months at $37^{\circ} \mathrm{C}$. Five loopfuls of the culture were removed from the surface of the medium and transferred to a screwcapped bottle containing glass beads. Five milliliters of sterile saline was added to the bottle and the contents shaken vigorously for 5 minutes to partially disperse the culture in the saline solution. The latter was then diluted further to $20 \mathrm{ml}$ with additional saline. One milliliter amounts of the bacterial suspension were added to a series of sterile, $15 \mathrm{ml}$ centrifuge

\footnotetext{
${ }^{1}$ Armour \& Company brand of a quaternary ammonium compound, tetradecylamine (see publication of C. R. Smith et al, The Bactericidal Effect of SurfaceActive Agents on Tubercle Bacill, Public Health Rep., No. 48, 1588-1600, Dec. 1, 1950).
} 
tubes. To each tube was then added $5 \mathrm{ml}$ of undiluted embalming solution. No attempt was made here to evaluate the solutions by dilution of the germicides in testing them against $M$. tuberculosis. The centrifuge tubes containing the organismgermicide solutions were allowed to stand at room temperature for three minutes at which time they were centrifuged for three minutes at $2000 \mathrm{rpm}$. The supernatant germicides

TABLE 1

Formulas of embalming solutions

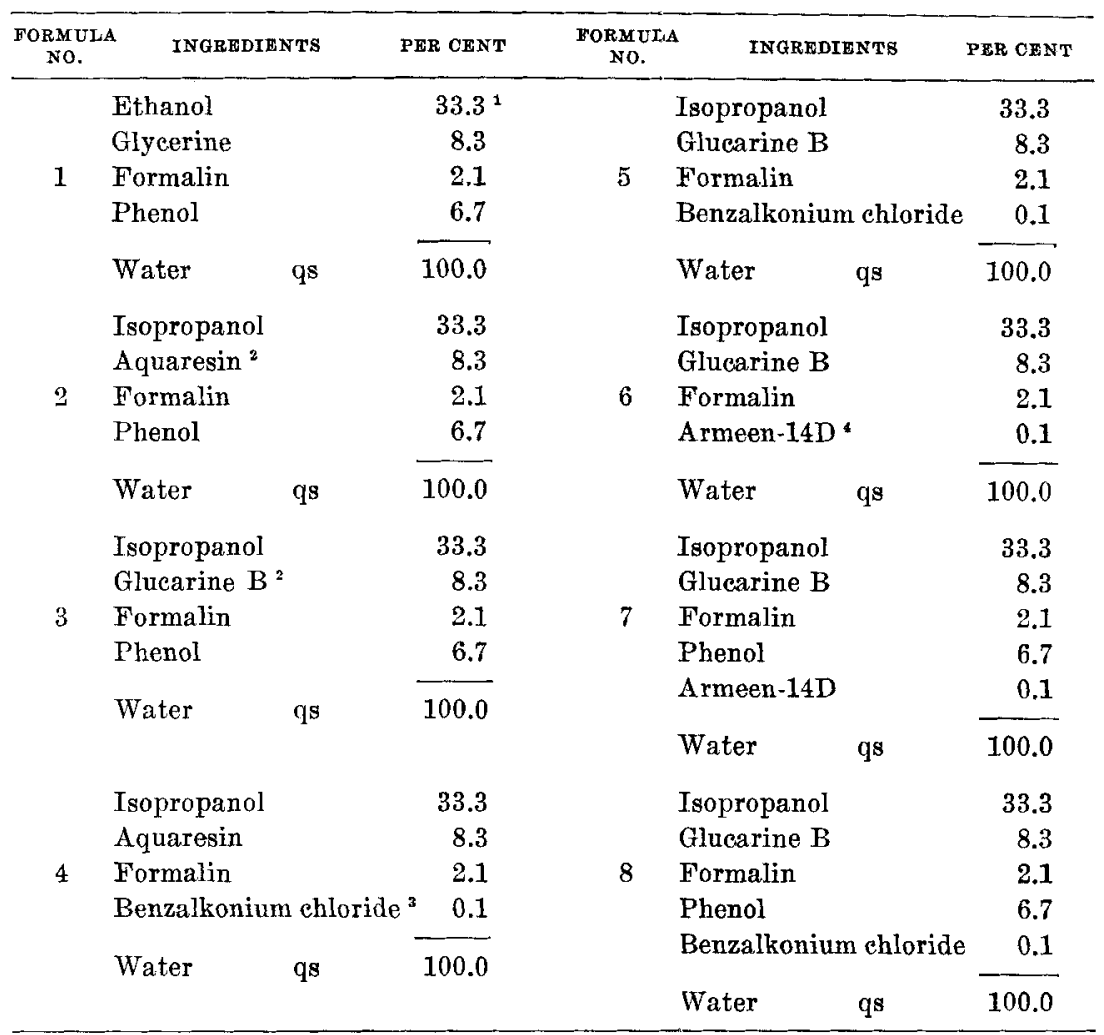

1 The precise decimal percentages are determined by the use of gallon measures in formulation by volumes and are not critical figures.

${ }^{2}$ Glyco Products Company brands of glycols.

${ }^{3} \mathrm{~A}$ quaternary ammonium surface-active germicide, alkyldimethylbenzylammonium chloride.

See footnote 1, page 508 . 
were removed from the packed cells by aspiration and the cells resuspended in sterile saline solution. The tubes were again centrifuged as before and the supernatant discarded. Two loopfuls of the packed cells were then transferred to each of two tubes of Petragnani's medium in screw-capped test tubes. The transplants were incubated at $37^{\circ} \mathrm{C}$. for three months before the final observations were made as to the presence or absence of growth of the acid-fast organisms.

Results. In all instances, no growth of the tubercle bacilli was noted in any of the transplant tubes. This indicates that all of the experimental formulations, including the original solution, destroyed the tubercle bacillus when the solutions were tested in the undiluted forms.

II. Germicidal action of embalming solutions on several non-sporulating bacteria. A study was made to determine the comparative antibacterial activities of the solution upon a Gram-positive organism, Staphylococcus aureus, and upon three Gram-negative bacteria, Eberthella typhosa, Pseudomonas aeruginosa and Proteus vulgaris. The germicidal solutions were diluted with distilled water and $5 \mathrm{ml}$ of the latter distributed in sterile culture tubes. Five-tenths milliliter amounts of 24-hour, undiluted beef extract broth cultures of the respective organisms were added to each of the diluted germicide solutions. The test suspensions were kept at room temperature $\left(23-26^{\circ} \mathrm{C}\right.$.) and at the end of exactly 5 minutes a $4 \mathrm{~mm}$ platinum loopful of the treated suspensions was transferred to a tube containing approximately $10 \mathrm{ml}$ of sterile beef extract broth. The latter transplants were incubated at $37^{\circ} \mathrm{C}$. for a period of 7 days at which time the presence or absence of visible growth was recorded. The results of this study are presented in table 2 .

Results. Examination of the data given in table 2 reveals the following pertinent points: (1) substitution of isopropanol for ethanol and Aquaresin or Glucarine B for glycerine (formulas 2,3) results in solutions with germicidal activity comparable to the original embalming solution (formula 1); (2) substitution of Benzalkonium chloride for the phenol 
in the solutions containing isopropanol and either of the substituted glycerine formulas (formulas 2,3 ) results in an enhancement of activity of the preparations (formulas 4,5 ); (3) substitution of the alternate quaternary ammonium compound, Armeen-14D, for phenol in the formula (in place of Benzalkonium chloride) does not show any increase in germicidal activity (formula 6); (4) the presence of phenol in the formula with Armeen-14D (formula 7) or of phenol with Benzalkonium chloride (formula 8) shows enhancement of activity over formula 6 ; (5) formula 8 shows the same degree of activity with respect to these organisms as the solution lacking the phenol (formula 5).

TABLE 2

Highest dilution of embalming solutions killing non-spore-forming organisms in 5 minutes at $23-26^{\circ} \mathrm{C}$.

\begin{tabular}{cllcc}
\hline \multirow{2}{*}{ solut Tion so. } & \multicolumn{3}{c}{ ORGANISM } \\
\cline { 2 - 5 } & S. aureus & E. typhosa & Ps. aeruginosa & Prot. vulgaris \\
\hline 1 & $1: 5$ & $1: 5$ & $1: 5$ & $1: 5$ \\
2 & $1: 5$ & $1: 5$ & $1: 5$ & $1: 5$ \\
3 & $1: 5$ & $1: 5$ & $1: 5$ & $1: 5$ \\
4 & $1: 20$ & $1: 20$ & $1: 10$ & $1: 10$ \\
5 & $1: 40$ & $1: 40$ & $1: 10$ & $1: 20$ \\
6 & $1: 5$ & $1: 5$ & $1: 5$ & $1: 5$ \\
7 & $1: 40$ & $1: 40$ & $1: 10$ & $1: 20$ \\
8 & $1: 40$ & $1: 40$ & $1: 10$ & $1: 20$ \\
\hline
\end{tabular}

With the evidence obtained in these preliminary tests, of the increased germicidal activity of salutions 5, 7 and 8 over the original and other experimental solutions, these preparations were studied further for their antimicrobial activity against three spore-forming bacteria, two Gram-positive pathogenic cocei, two non-pathogenic and three pathogenic fungi.

III. Germicidal action of embalming solutions on several pathogenic spore-forming bacilli and Gram-positive cocci. Dilutions of the original embalming solution and the three more active experimental formulations (formulas $5,7,8$ ) were 
prepared as indicated before. The amount of bacterial inoculum, temperature of treatment, and time of exposure were also the same. Bacillus anthracis was grown and transplanted in beef extract medium. The two pathogenic, anaerobic clostridia, Cl. tetani and $\mathrm{Cl}$. novyi, were grown and transplanted in Brewer's thioglycollate medium. Streptococcus pyogenes (beta hemolytic) and type I pneumococcus were grown and transplanted in Brain Heart Infusion (Difeo) broth. The transplants were incubated at $37^{\circ} \mathrm{C}$. and the presence or absence of visible growth recorded at the end of 7 days. The results of this experiment are presented in table 3.

TABLE 3

Highest dilution of embalming solutions killing spore-forming bacilli and Gram-positive cocei in 5 minutes at $23-26^{\circ} \mathrm{C}$.

\begin{tabular}{|c|c|c|c|c|c|}
\hline \multirow{2}{*}{$\begin{array}{c}\text { SOLUTION } \\
\text { NO. }\end{array}$} & \multicolumn{5}{|c|}{ ORGANISM } \\
\hline & B. anthracis & Cl. tetani & Cl. novyi & Strep. pyogenes & $\begin{array}{c}\text { Type I } \\
\text { pneumo. }\end{array}$ \\
\hline 1 & $1: 10$ & $1: 10$ & $1: 10$ & $1: 5$ & $1: 10$ \\
\hline 5 & $1: 80$ & $1: 40$ & $1: 40$ & $1: 80$ & $1: 160$ \\
\hline 7 & $1: 40$ & $1: 160$ & $1: 20$ & $1: 40$ & $1: 40$ \\
\hline 8 & $1: 40$ & $1: 160$ & $1: 80$ & $1: 80$ & $1: \mathbf{1 6 0}$ \\
\hline
\end{tabular}

Results. From the data given in the table it is evident that all three experimental solutions are superior in germicidal activity to the original embalming solution. It is also apparent that differences in activity exist among the three experimental formulations. Nevertheless, all were included, along with the original solution, in a study of their antifungal activity.

IV. Action of embalming solutions on fungi. The selected germicides were diluted in distilled water as indicated previously and tested against two non-pathogenic fungi, Penicillium notatum and Aspergillus niger, and three pathogenic fungi, Coccidioides immitis, Histoplasma capsulatum and Cryptococcus neoformans. In all instances the fungi were grown on Sabouraud's maltose agar slants for 10-14 days. Sterile saline was added to the tubes and the growths removed from the medium as much as possible with a sterile platinum 
loop. The heterogenous suspensions were then filtered through sterile gauze and the material diluted further with sterile saline solution to provide sufficient culture suspensions to be used in the tests. Five-tenths milliliters of the cultures were added to each $5 \mathrm{ml}$ amounts of the germicidal solutions. Transplants were made at exactly 5 minutes to $10 \mathrm{ml}$ amounts of sterile liquid medium consisting of $4 \%$ maltose and $1 \%$ Neopeptone (Difco) in distilled water. The transplants were incubated at $37^{\circ} \mathrm{C}$ for three weeks at which time the presence or absence of visible growth in the tubes were recorded. The results of the fungicidal tests are presented in table 4.

TABLE 4

Highest dilution of embalming solutions killing fungi in 5 minutes at $29-26^{\circ} \mathrm{C}$.

\begin{tabular}{|c|c|c|c|c|c|}
\hline \multirow{2}{*}{$\begin{array}{l}\text { SOLUTION } \\
\text { No. }\end{array}$} & \multicolumn{5}{|c|}{ ORGANISM: } \\
\hline & Pen. notrtum & Asp. niger & C. immitis & H. capsulatum & C. neoformans \\
\hline 1 & $1: 5$ & $<$ undil. $^{1}$ & $1: 40$ & $1: 10$ & $1: 10$ \\
\hline 5 & undil. & $<$ undil. $^{1}$ & $1: 40$ & $1: 20$ & $1: 20$ \\
\hline 7 & $1: 5$ & undil. & $1: 80$ & $1: 40$ & $1: 40$ \\
\hline 8 & $1: 5$ & undil. & $1: 80$ & $1: 40$ & $1: 80$ \\
\hline
\end{tabular}

${ }^{1}$ Indicates that the undiluted solution failed to destroy the organisms and thus growth appeared in the transplant medium.

Results. From the data given in the table it is evident that formulations 7 and 8 are more active as fungicidal agents than solution 5 or the original formula (formula 1 ). The overall greater effectiveness of formula 8, with respect to certain of the pathogenic organisms tested (see also table 3), led to its selection for cadaver use.

\section{DISSECTING LABORATORY TRIAL}

The formula adopted (number 8 ) has been used in routine embalming and has not exhibited any particular difficulties or problems of handling in that procedure. In the dissection rooms the result has also been highly satisfactory. The bodies are relatively soft and plastic and the joints move with ease. The color of muscle is a medium brown, not too bleached 
although not the natural color of muscle. There is good differentiation of fascia, fat, nerve, tendon and other components of the tissues of the body. There is a somewhat different odor due to the use of isopropanol, but it is not objectionable; in fact, students using bodies containing the isopropanol express a preference for its odor. On almost all counts of comparison the new formula produces a more satisfactory embalming of the cadaver than the old.

With respect to the cost of the elements of this new formulation, it became apparent that a definite monetary saving occurs in the utilization of materials not necessarily inferior in quality but less costly because of a less competitive position with respect to industrial usage. The cost of isopropanol in this locality is approximately $60 \%$ that of ethanol, and the cost of Glucarine B approximates $65 \%$ that of glycerine. Benzalkonium chloride comes in a $50 \%$ solution and costs slightly in excess of $\$ 6.00$ a gallon, but in the percentage used a gallon of this material covers a considerable number of bodies. When costs of the various elements are computed on drum or quantity purchases, the embalming fluid figures out to approximately 60 cents a gallon in this locality. In terms of general cost operation this would appear to be an entirely reasonable figure.

\section{SUMMARY AND CONCLUSION}

1. An improved embalming fluid formula has resulted from a study of various ingredients as to cost and effectiveness.

2. The substitution of isopropanol for ethanol and the use of a substitute for glycerine results in a considerable decrease in cost without diminution of germicidal effectiveness.

3. The addition of a quaternary ammonium surface-active germicide enhances materially the effectiveness of the solution.

4. Laboratory observations indicate that the formula adopted is superior to previously used embalming fluids. 